\title{
Extraction of Knowledge Rules for the Retrieval of Mesoscale Oceanic Structures in Ocean Satellite Images
}

\author{
Eva Vidal-Fernández, Jesús M. Almendros-Jiménez, \\ José A. Piedra and Manuel Cantón
}

\begin{abstract}
The processing of ocean satellite images has as goal the detection of phenomena related with ocean dynamics. In this context, Mesoscale Oceanic Structures (MOS) play an essential role. In this chapter we will present the tool developed in our group in order to extract knowledge rules for the retrieval of MOS in ocean satellite images. We will describe the implementation of the tool: the workflow associated with the tool, the user interface, the class structure, and the database of the tool. Additionally, the experimental results obtained with the tool in terms of fuzzy knowledge rules as well as labeled structures with these rules are shown. These results have been obtained with the tool analyzing chlorophyll and temperature images of the Canary Islands and North West African coast captured by the SeaWiFS and MODIS-Aqua sensors.
\end{abstract}

Keywords Remote sensing - Satellite images - Mesoscale oceanic structures • Image processing $\cdot$ Tools $\cdot$ SeaWiFS $\cdot$ MODIS-Aqua

\section{Introduction}

With the increasing in the last decades of environmental problems like the global climate change [1-3] and the ocean primary production changes [4], the development of tools focused on intelligent search of objects and regions in satellite images stored in large databases of spatial and environmental agencies, should be a major goal. In this context, the global oceanic circulation plays an essential role in the global climate, because the ocean covers more than $70 \%$ of the surface of our planet. Most of the oceanic circulation is mesoscale (i.e., scale from 50 to $500 \mathrm{~km}$ and $10-100$ days), and the energy of Mesoscale Oceanic Structures (MOS) is at least one order of magnitude greater than the general circulation. Thus, MOS are crucial for ocean dynamic study and global change analysis $[5,6]$. 
The main types of MOS are upwellings, upwelling filaments, cold/warm eddies, and wakes [7-11]. A coastal upwelling can be defined as the interaction between the bottom and surface water. upwellings are mainly due to oceanic currents which are, at the same time, influenced by wind dynamics. They occur when dense cold water at the bottom of the ocean rises to the surface near the coast, transporting nutrients, and enabling the development and proliferation of phytoplankton [12]. These upwellings occur regularly (during the whole year with varying intensity) along the North West African coast (see Fig. 1) and others like the Peruvian, Californian and South African coasts, among others, where the wind conditions are suitable [1, 3, 13-16]. The analysis and prediction of upwellings, for which satellite images are a powerful tool, are very important to commercial fishing. There are some mesoscale cross-shore structures along the upwelling front called upwelling filaments which are tongue-shaped cold upwellings. They are important nutrient carriers from coast to the open ocean, often found near capes [17] (e.g., off Cape Ghir or Cape Blanc) (see Fig. 1). Eddies are highly morphological and contextual variable structures $[8,11]$. They may appear rounded near islands or in the open ocean. In cool eddies, cold nutrient-rich water rises to the surface [18], while warm eddies (see Fig. 1) drag water with organic material to the ocean floor and keep in the warm surface. eddy water differs from the surrounding water in salinity and temperature, and can also travel long distances for long periods of time without mixing with the surrounding

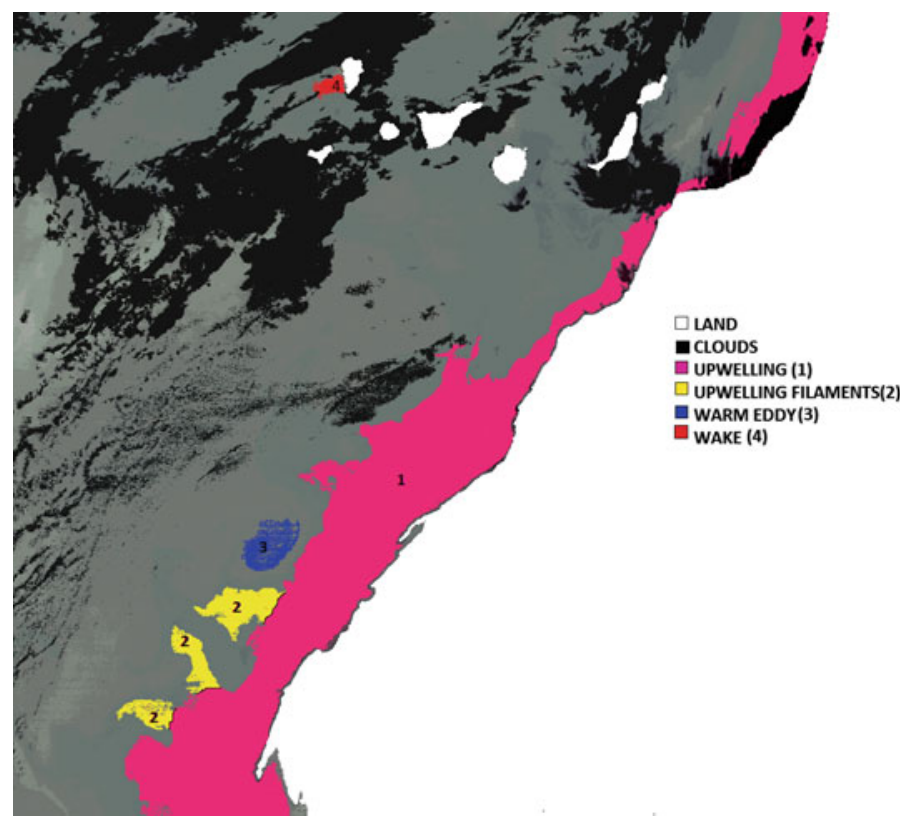

Fig. 1 MODIS-Aqua-Sea surface temperature (SST) scene (2003-03-24). Ocean structure map where the upwelling has been colored in pink for visualization (1), upwelling filaments (Cap Blanc) in yellow (2), warm in blue (3), and wake (west of La Palma) in red (4) 
water $[12,19]$. The cool (cyclonic) eddies are created by calm wind conditions, while warm (anticyclonic) eddies are formed under stronger wind conditions. Wakes are warm oceanic structures associated with islands $[12,20]$, and can be created by obstacle formed by islands. This interaction causes very thin tongues of hot, nutrient poor water (as compared to the surrounding water) to form close to the southwest islands (see Fig. 1). These structures are shown in Fig. 1.

The images used in our analysis correspond to a wide temporal range (from 1997 to 2013, and for each month), with clouds partially hiding structures, and as consequence MOS are very different from one time to another. Even when region recognition is automatic (by image segmentation), there are some factors that difficult MOS detection. Some factors are the presence of noise and clouds, as well as regions with holes, and regions which cannot be distinguished from the surrounding water (due to their size or their color). To overcome some of these handicaps, the initial color scale used in the image processing stage can be modified. To overcome the handicap of clouds and noise, which split a region into small regions, regions can be merged.

\subsection{Contributions of the Chapter}

In this chapter we will describe the implementation of a tool for the extraction of fuzzy knowledge rules. Such knowledge rules are required to retrieve regions from the tool database in order to be labeled. knowledge rules can thus considered as queries against the database. The experimental results obtained with the tool in terms of knowledge rules as well as labeled structures with these rules are shown. These results have been obtained with the tool analyzing chlorophyll and temperature images of the Canary Islands and North West African coast captured by SeaWiFS (Seaviewing Field-of-view Sensor) and MODIS-Aqua (Moderate Resolution Imaging Spectroradiometer, on board the Aqua satellite) sensors. The tool will be described as follows:

1. Workflow for Labeling MOS: The workflow we present in this chapter describes the steps required to label a MOS. It provides a number of steps to be followed by the oceanographer in order to label a MOS in reasonable time. Following these steps a novice user of the tool would be able to label MOS. Nevertheless, the needed time depends on the image characteristics. For instance, whether the image includes more than one MOS, whether MOS are divided in pieces due to clouds, etc. The workflow shows a complete case distinction, enabling to get good results even for novice users.

2. Extraction of knowledge rules: A method for extraction of knowledge rules is presented. The core of the workflow for labeling MOS is the extraction of rules. These knowledge rules are described in terms of fuzzy concepts, and the method for extraction is a trial and error procedure which refines knowledge rules in each step from an initial test. 
3. Experimental Results: The dataset used to test our tool consists on MOS described by 64 image descriptors. The experimental results provide knowledge rules which make possible to retrieve and label 365 MOS. The knowledge rules are built from fuzzy features (15 from the initial 64 features). In some cases, some regions have been merged to get a compact and well-contoured MOS. In particular, the tool enables to manually merge regions, using the so-called ROI (Region of Interest). Experimental results are described by number and type of MOS, whether or not ROI is used, as well as the knowledge rules for each case.

\subsection{Related Work}

In our approach primary objects (output of the segmentation phase) evolve toward objects of interest (regions), with the help of the knowledge of an expert (oceanographer) [21] and specific techniques of segmentation for each type of object [22]. It is crucial in the context of MOS, which are characterized by high morphological and contextual variability [23-25]. Some examples of techniques and tools for region retrieval from images can be found in [26-29].

Our research group has a wide experience in the development of methods and techniques for the analysis of ocean satellite images, mainly focused on sensors capturing the area of study (i.e., Canary Islands and North West African coast) (see [28-32], for the most recent contributions). In particular, the same dataset (and thus of the same sensor) was used in two previous works [28, 29]. The tool we present here has permitted the automatization of the process of region labeling and merging, and the automatization of knowledge rule extraction. This is crucial in a high morphological and contextual variability context, in which a trial and error method has to be used to detect MOS. In [29], we had the same problems of MOS identification: presence of noise and clouds, as well as regions with holes, and regions which cannot be distinguished from the surrounding water. We already there modified the initial color scale to get well-contoured and compact regions from segmentation. However, we found there that it can be still not enough, and manual merging is required. The tool we present here is able to automatically merge regions from manual selection of the ROI by the expert. Thus manual (although assisted by the tool) merging can be done now. One the other hand, the definition of knowledge rules is hard when it is manually done. The tool enables to try knowledge rules playing from the user interface with several choices of fuzzy descriptors and range of values, and more interesting to compare with previously labeled regions and previously defined knowledge rules, which reduces significantly the time required to define new knowledge rules. Thus, the development of the tool has permitted to reduce drastically the time for labeling and extraction of knowledge rules, and also reuse, detection of patterns, etc. The tool (i.e., workflow, procedure of knowledge rule extraction and image comparison) is the main contribution of this work, and in order to validate the tool, we will show some examples of images in which the manual merging is required, and report experimental results of manual merging. The number and type 
of knowledge rules, involving fuzzy descriptors and values, is also of great interest for oceanographers interested in the analyzed area.

\subsection{Structure of the Chapter}

The structure of the chapter is as follows. Section 2 will describe the dataset. Section 3 will show the workflow of the tool. Section 4 will present the knowledge rules and labeling results obtained from the analyzed images. Section 5 will describe the implementation of the tool. Finally, Sect. 6 will conclude and present future work.

\section{Dataset}

We have analyzed 212 satellite images of the Canary Islands and North West African coast captured by SeaWiFS sensor on board the Orbview- 2 satellite $(1.1 \mathrm{~km}$ resolution) and MODIS-Aqua sensor on board the Aqua satellite (1.1 km resolution), obtained from the Ocean Color Web of NASA [33]. Many studies have reveal that SeaWiFS and MODIS-Aqua-OC sensor chlorophyll images and MODIS-Aqua-SST temperature sensor images are a suitable choice for the study of MOS [34-37], primary production [38], and global change [39]. In [40] the authors analyze data from chlorophyll images of a 10-year period, comparing them with in situ collected data. They also compare results with different sensors, finding highly correlated coefficients. A total of 212 images have been analyzed. The number of processed images was larger, but most of them were discarded by the tool due to several reasons: too many clouds, out of the study area, faulty images, etc. Of them, 92 images are chlorophyll images acquired from the SeaWiFS sensor, from 1997 to 2004, and covering all the seasons (9-1997; 14-1998; 25-1999; 14-2000; 7-2001; 5-2002; 7-2003, and 11-2004). These images are derived from MLAC (Merged Local Area Coverage product) products. The MLAC products consolidate all of the LAC from different receiving stations available for the same orbit in geographic regions with multiple HRPT (High Resolution Picture Transmissions) stations. Overlapping scenes are evaluated to acquire a single best image without duplication. The MLAC product was chosen because it generates high-quality L2 level images, including radiometric calibration and geometric correction. The other 120 images are from the MODISAqua sensor, for 2003-2013 and divided into 61 chlorophyll and 59 SST images. These images are derived from L2 level LAC (Local Area Coverage) products.

In the case of MODIS sensor, we worked with two products: those about both chlorophyll (OC) and temperature (SST), and thus providing two images, and those about only chlorophyll or only temperature, from which a single image is obtained. This is the reason why the number of chlorophyll images is larger than the number of temperature images for products L2 Level LAC of MODIS. We analyzed in terms of years: 2003-2 OC and 2 SST; 2004-5 OC and 4 SST; 2008-19 OC and 19 SST; 
2009-12 OC and 13 SST; 2010-10 OC and 16 SST; 2011-4 OC and 1 SST; 2012-6 OC and 3 SST; and 2013-3 OC and 1 SST, covering all the seasons.

Therefore, a total of 212 satellite images of the Canary Islands and the Northwest African coast have been analyzed, where a large number of MOS can be found. A total of 365 Mesoscale Oceanic Structures were detected of which 284 MOS correspond to upwellings or parts of upwellings (when clouds keep parts upwellings from being merged), 44 upwelling filaments, 10 cool eddies, four warm eddies and 23 wakes.

\section{Workflow of the Tool}

Figure 2 shows the steps (from 1 to 20 ) to be followed by an user of tool. The first step consists of the selection of (1) color table (chlorophyll or temperature) and (2) images to process. The color scale is represented by a table in which each entry is an assignment of a color range of Chl-a (i.e., chlorophyll concentration of the sea surface) or SST (i.e., Sea Surface temperature) to a given color. In such a way that each value of the HDF (Hierarchical Data Format) matrix is converted into a colored pixel. HDF files include several groups of data. One of the groups contains geophysical data with matrices of Chl-a, SST and L2-flags. In order to convert HDF values into colored pixels, for each pixel of the Chl-a (or SST) matrix, the flag 32 is used to know whether the value is correct or wrong. In the case the value is correct, the flag 2 is used to know whether it belongs to land. In case it is correct and not a land value, the value of the Chl-a (or SST) matrix reports the value of the color table.

The choice of a suitable clustering of colors (i.e., color table) for a given image, enables better recognition of regions: well-contoured and compact regions and regions obtained from several small regions of almost identical color. In this step, starting from the initial color table (see Table 1 for chlorophyll), colors are grouped (although not necessarily). For instance, in Table 2 the color clustering "Red to Yellow" is shown. Such a color clustering means that colors from yellow and beyond are grouped and identified to "Red". Similar tables for temperature images can be also considered.

Figure 3 shows the processing result of an image of SeaWiFS (i.e., chlorophyll) using the original table, and the color clustering "Red to Yellow". The "Red to Yellow" color clustering permits the recognition of well-contoured and compact regions without holes, facilitating MOS detection and labeling. In this image, a big upwelling from Canary Islands to Cape Blanc and a cold eddy in the south of Gran Canaria island are detected and showed in red.

After steps (1) and (2) (selection of color tables and images) processing is carried out (3). This automatic task involves several subprocesses: decompression and extraction of the HDF file content: the matrix of chlorophyll data (Chl-a) or temperature data (SST), transformation to a color image according to the color table, and segmentation (combining smoothing filter with thresholding techniques and edge detection). Additionally (spectral, morphologic and contextual) descriptors of regions are computed. 


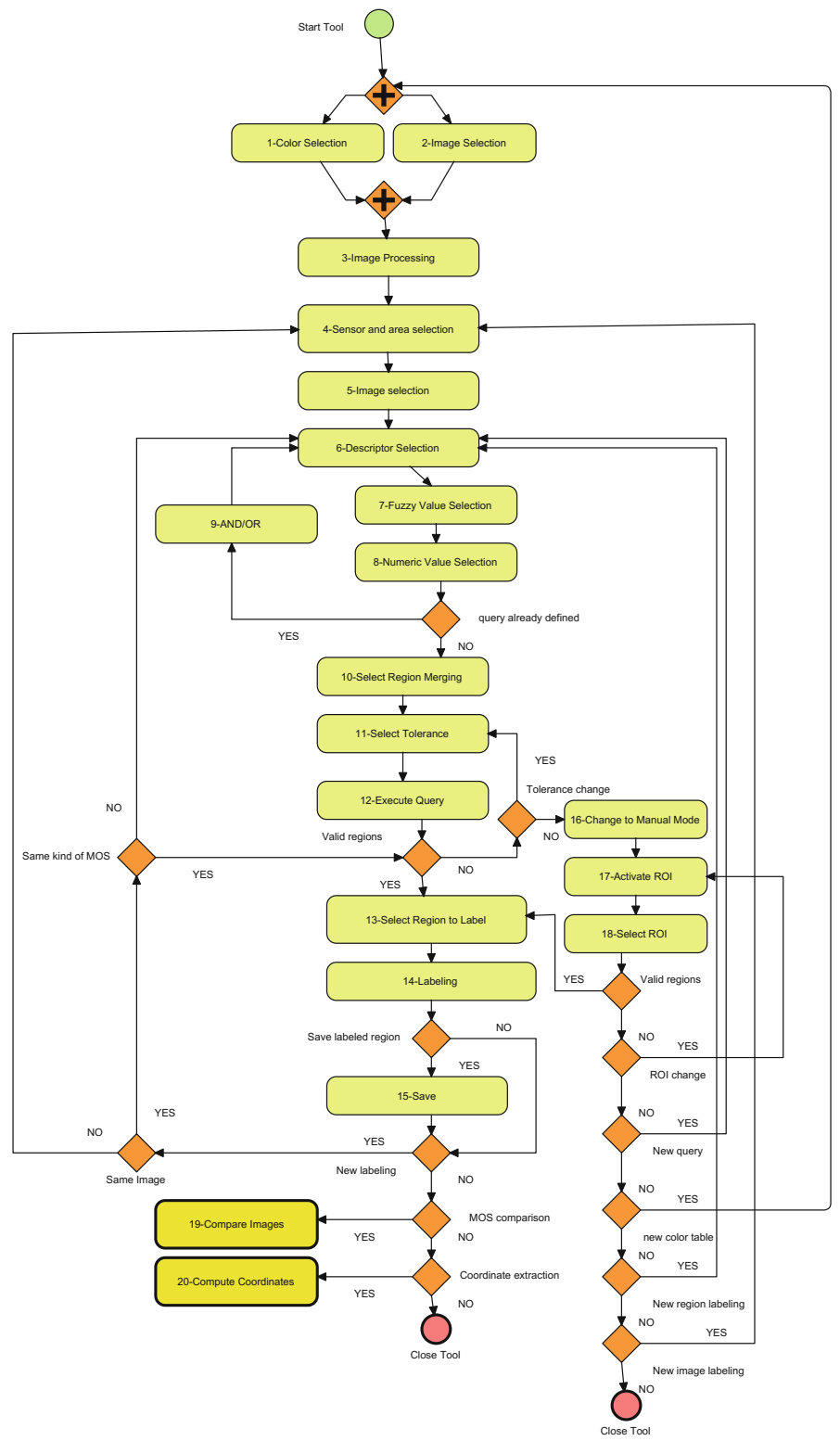

Fig. 2 Workflow of the tool 
Table 1 Original SeaWiFS and MODIS sensor OC image color table

\begin{tabular}{l|l|l|l}
\hline RGB Color & Color & Name & Chl-a $\left(\mathrm{mg} / \mathrm{m}^{3}\right)$ \\
\hline $1 ; 1 ; 223$ & $\square$ & Dark blue & $0.03-0.06$ \\
\hline $46 ; 46 ; 254$ & $\square$ & Blue & $0.06-0.1$ \\
\hline $88 ; 250 ; 244$ & $\square$ & Turquoise & $0.1-0.2$ \\
\hline $129 ; 247 ; 243$ & $\square$ & Light blue & $0.2-0.3$ \\
\hline $46 ; 254 ; 46$ & $\square$ & Green & $0.3-0.6$ \\
\hline $129 ; 247 ; 129$ & & Light green & $0.6-1$ \\
\hline $244 ; 250 ; 88$ & & Light Yellow & $1-2$ \\
\hline $255 ; 255 ; 0$ & $\square$ & Yellow & $2-3$ \\
\hline $254 ; 154 ; 46$ & & Light orange & $3-5$ \\
\hline $255 ; 128 ; 0$ & & Orange & $5-7$ \\
\hline $254 ; 46 ; 46$ & & Light red & $7-10$ \\
\hline $255 ; 0 ; 0$ & & Red & $10-100$ \\
\hline
\end{tabular}

Table 2 Example of color clustering: from light yellow to red

\begin{tabular}{l|l|l|l}
\hline RGB color & Color & Name & Chl-a $\left(\mathrm{mg} / \mathrm{m}^{3}\right)$ \\
\hline $1 ; 1 ; 223$ & $\square$ & Dark Blue & $0.03-0.06$ \\
\hline $46 ; 46 ; 254$ & $\square$ & Blue & $0.06-0.1$ \\
\hline $88 ; 250 ; 244$ & & Turquoise & $0.1-0.2$ \\
\hline $129 ; 247 ; 243$ & & Light blue & $0.2-0.3$ \\
\hline $46 ; 254 ; 46$ & & Green & $0.3-0.6$ \\
\hline $129 ; 247 ; 129$ & & Light Green & $0.6-1$ \\
\hline $255 ; 0 ; 0$ & $\square$ & Red & $1-2$ \\
\hline $255 ; 0 ; 0$ & - & Red & $2-3$ \\
\hline $255 ; 0 ; 0$ & - & Red & $3-5$ \\
\hline $255 ; 0 ; 0$ & - & Red & $5-7$ \\
\hline $255 ; 0 ; 0$ & & Red & $7-10$ \\
\hline $255 ; 0 ; 0$ & & Red & $10-100$ \\
\hline
\end{tabular}


(a)

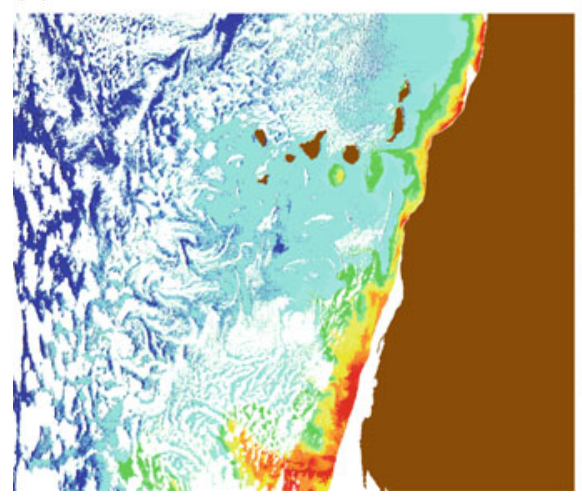

(b)

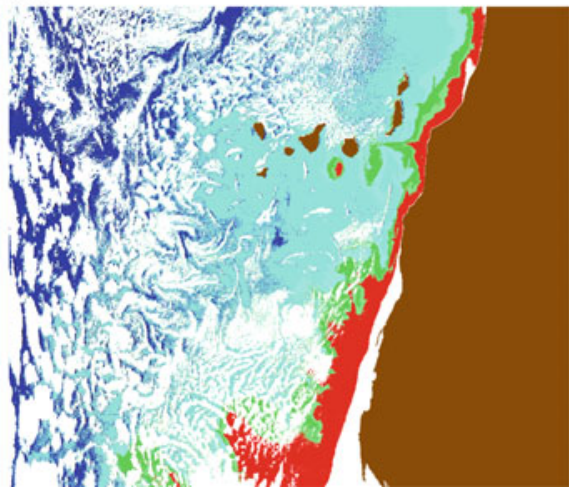

Fig. 3 SeaWiFS scene (2004-07-09). a Result of processing with original color table. b Result of processing with Yellow to Red color table

MOS labeling is the next step. It also involves two steps: sensor selection (i.e., SeaWiFS, MODIS-Aqua-OC or MODIS-Aqua-SST) and earth area selection (i.e., Canary Islands) (4) and image selection (5). In previous tasks (i.e. (1) (2) and (3)) more than one image can be processed, and now selection of earth area and sensor filters from the batch of processed images.

\subsection{Extraction of Knowledge Rules}

After the selection of the image to be labeled, the oceanographer has to define the knowledge rule for the retrieval of the MOS. The extraction of knowledge rules involves the selection of fuzzy spectral, morphologic and contextual features (6) (i.e., size, island distance, continent distance, temperature and chlorophyll concentration), the fuzzy value (7) (i.e., near, medium and far for distances, small, medium and big for size, very warm, warm, temperate, cold and very cold for temperature, and very low, low, medium, high and very high for concentration) and the range of the fuzzy numeric value ( 8 ) from zero to one (for instance, $\geq 0.9$ and $<0.5$ ). The tool works with fuzzy membership functions, for spectral, morphologic and contextual features, given by triangular functions, in such a way that a membership degree is assigned to each fuzzy value. The goal of the oceanographer is to select, for instance, those regions whose membership degree to a given fuzzy value of size, for instance, big, is greater or equal than a given value, for instance, 0.9 ; in other words, the oceanographer wants to retrieve a big region. Analogously, the oceanographer can select regions whose membership to the fuzzy value warm is greater or equal than 0.7 ; in other words, the oceanographer wants a warm region. The oceanographer can add several 
Boolean conditions (9) of this type, in such a way that a query can be considered as a knowledge rule, with ANDs and ORs.

The method for extraction is a trial and error procedure which refines knowledge rules in each step from an initial test. The initial test and refinements take into account the particular MOS characteristics (upwelling, wake, eddy) as well as the image characteristics (temperature, chlorophyll), as well as the time of capture (cold or warm water, presence of clouds, etc.). The best strategy is to select limit values of fuzzy ranges (for instance, high concentration of chlorophyll in case of upwelling) and to refine the rule adding new fuzzy descriptors (size or distance), until a complete MOS is retrieved.

When the knowledge rule has been defined, the oceanographer can select automatic region merging (10) according to a given tolerance level (11). The merging algorithm based on a technique of region growing, unifies regions of similar properties, whenever they are separated by a given distance, called tolerance, measured by pixels. The merging algorithm has been designed in order to merge regions according to fuzzy knowledge rules. The algorithm takes as input a set of regions and returns a merged region. Two conditions are simposed for region merging: regions satisfy the same fuzzy knowledge rule and regions are closed. The algorithm uses a ROI (region of interest), which is a rectangle enclosing the region. In order to merge regions, the regions should be closed and thus the ROIs of the regions should intersect. The algorithm builds a new ROI each time two regions are merged, and the algorithm iterates over the set of regions until a unique ROI has been built. In case a ROI of a region does not intersect with the computed ROI the region is discarded. The algorithm has a parameter called tolerance allowing a weaker intersection of ROIs. By default, tolerance is 0 , but it can be customized from 0 to 20 .

Once the knowledge rule is executed (12), a list of regions are retrieved (some of them are merged regions whenever the automatic merging mode has been selected). The list includes simple and merged regions. Now, the oceanographer has to answer the following question: Are the regions valid? In the positive case, the oceanographer selects the region (simple or merged) to be labeled (13), and the oceanographer proceeds to label (15). In Fig. 4a an example of labeled region (a pink upwelling) is shown. The tool permits to save the labeled image (15), to label another MOS of the same image, to label a new image, and to compare labeled regions of other images (19) or the same image. Additionally, the coordinate matrix of the processed image can be computed and visualized (20).

In the negative case (i.e., regions are not valid), the oceanographer can go back and change tolerance. When tolerance change does not give a better result, the oceanographer can select manual mode. Manual mode (16) permits to activate and use a ROI (i.e., region of interest) (17), which is a rectangle, making possible to select more than one region of the image. With manual mode, regions can be forced to be merged and unified. The ROI selection (i.e., the regions) is manual, but merging is automatic. In Fig. $4 \mathrm{~b}$ a cold eddy is shown in pink color. This image is the same as the image of Fig. 4a, but the eddy of Fig. 4b in pink color is bigger than the eddy of Fig. 4a in red color. 
(a)

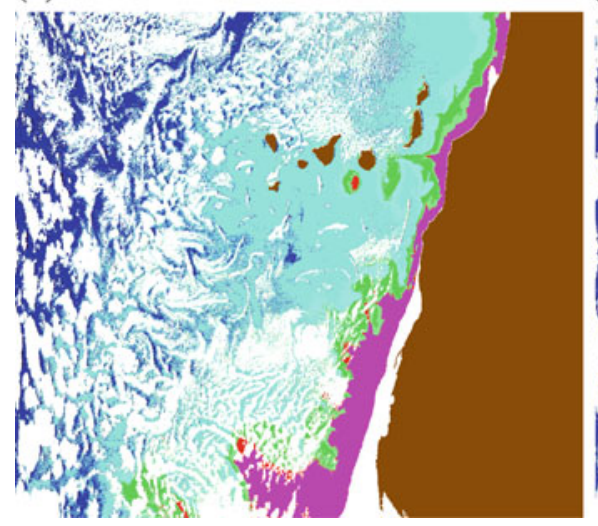

(b)

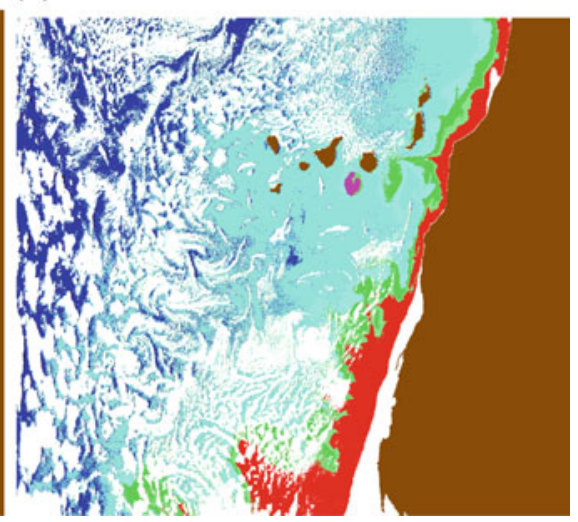

Fig. 4 SeaWiFS scene (2004-07-09). a Upwelling labeled in pink color. b Cold eddy labeled in pink color. Manual mode

When manual mode does not improve the result, the oceanographer has to go back to knowledge rule definition (redefining or modifying the knowledge rule), or to image processing (selecting a new color table).

\section{Experimental Results}

In this section, we report the results obtained with our tool in terms of knowledge rules and labeled structures for SeaWiFS and MODIS-Aqua images. On one hand, we show knowledge rules for the identification of MOS in these images. One the other hand, type and number of structures for each class of image are given.

\subsection{Knowledge Rules}

31 optimal and valid knowledge rules ( 12 chlorophyll rules and 19 temperature rules) have been obtained from 212 processed images and 365 labeled MOS. Table 3 shows 10 basic knowledge rules for chlorophyll (SeaWiFS and MODIS-Aqua-OC). They can be used alone, or in combination with other, in total 12, shown in Table 4. The chlorophyll images have been processed using the color clustering "Red to Yellow," the best for MOS detection and labeling for chlorophyll images.

These knowledge rules have been applied to 153 chlorophyll images, labeling all the MOS of the images. In some cases, manual mode is used. Next, we specify which rules are used in each case, and the needed for ROI in each case. The most 
Table 3 Basic knowledge rules of chlorophyll (AND rules). Ch: chlorophyll; CD: Continent Distance; ID: Island Distance; VL: Very Low (Fuzzy_value_1); L: Low (Fuzzy_value_2); M: Medium (Fuzzy_value_3); H: High (Fuzzy_value_4); VH: Very High (Fuzzy_value_5); N: near; M: medium; F: far; S: small; $\mathbf{M}$ : medium; B: big

\begin{tabular}{l|l|l|l|l|l|l|l|l|l|l|l|l|l|l}
\hline \multirow{2}{*}{ Rule } & \multicolumn{9}{l}{ Ch } & \multicolumn{9}{l}{ CD } & \multicolumn{1}{l|}{ ID } & \multicolumn{3}{l|}{ Size } & \\
& VL & L & M & H & VH & N & M & F & N & M & F & S & M & B \\
\hline C1 & & & & & $\geq 0.25$ & $\geq 0.5$ & & & & & & & & \\
\hline C2 & & & $\geq 0.5$ & & & $\geq 0.5$ & & & & & & & & \\
\hline C3 & & & $\geq 0.25$ & & & $\geq 0.5$ & & & & & & & & \\
\hline C4 & & & & & $\geq 0.25$ & $\leq 0.5$ & & & & & & & & \\
\hline C5 & & & $\geq 0.5$ & & & $\leq 0.5$ & & & & & & & & \\
\hline C6 & & & $\geq 0.25$ & & & $\leq 0.5$ & & & & & & & & \\
\hline C7 & & & & & $\geq 0.25$ & & & & & & & & & \\
\hline C8 & & & $\geq 0.25$ & & & & & & & & & & & \\
\hline C9 & $\geq 0.1$ & & & & & & & & & & & & & \\
\hline C10 & $\geq 0.1$ & & & & & & & & $\geq 0.5$ & & & $\geq 0.4$ & & \\
\hline
\end{tabular}

Table 4 Knowledge rules

\begin{tabular}{|c|c|c|c|c|c|}
\hline \multicolumn{2}{|c|}{ Knowledge rules } & \multicolumn{2}{|c|}{ Chlorophyll Rules (CR) } & \multicolumn{2}{|c|}{ Temperature Rules (TR) } \\
\hline \multicolumn{2}{|c|}{ CR } & \multicolumn{2}{|c|}{ TR } & \multicolumn{2}{|c|}{ TR } \\
\hline $\mathrm{RC} 1$ & $\mathrm{C} 1$ & RT1 & $\mathrm{T} 1$ & RT10 & $\mathrm{T} 8$ \\
\hline $\mathrm{RC} 2$ & $\mathrm{C} 1 \mathrm{OR} \mathrm{C} 2$ & RT2 & $\mathrm{T} 2$ & RT11 & T9 \\
\hline $\mathrm{RC} 3$ & $\mathrm{C} 1 \mathrm{OR} \mathrm{C} 3$ & RT3 & T3 & RT12 & T10 \\
\hline $\mathrm{RC} 4$ & $\mathrm{C} 4$ & RT4 & $\mathrm{T} 4$ & RT13 & T11 \\
\hline RC5 & C4 OR C5 & RT5 & $\mathrm{T} 5$ & RT14 & T12 \\
\hline RC6 & C4 OR C6 & RT6 & T1 OR T5 & RT15 & T13 \\
\hline $\mathrm{RC7}$ & $\mathrm{C} 5$ & RT7 & T1 OR T4 & RT16 & T14 \\
\hline RC8 & C6 & RT8 & T2 OR T6 & RT17 & T15 \\
\hline RC9 & $\mathrm{C} 7$ & RT9 & T2 OR T7 & RT18 & T16 \\
\hline $\mathrm{RC} 10$ & C7 OR C8 & \multirow[t]{3}{*}{ RT19 } & \multirow[t]{3}{*}{$\mathrm{T} 17$} & \multirow[t]{3}{*}{ RT19 } & \multirow[t]{3}{*}{ T17 } \\
\hline $\mathrm{RC} 11$ & C9 & & & & \\
\hline $\mathrm{RC} 12$ & $\mathrm{C} 10$ & & & & \\
\hline
\end{tabular}

used descriptors are, obviously, those for chlorophyll concentration, near continent distance as well as near island distance, according to MOS definitions.

For temperature images, the original color table is used because a color clustering suitable for all the cases cannot be found. It is due since there are scenes from several times of year, and temperature considerably varies from one time to another. We took this decision to have a criterion for comparison. Testing images individually, a color clustering suitable for surrounding water temperature, makes possible more compact and well-contoured regions (without merging), enabling recognition and 
Table 5 Basic knowledge rules for temperature (AND rules). T: temperature; CD: Continent Distance; ID: Island Distance; VC: Very Cold (Fuzzy_value_1); C: Cold (Fuzzy_value_2); M: Medium (Fuzzy_value_3); W: Warm (Fuzzy_value_4); VH: Very Hot (Fuzzy_value_5); N: near; M: medium; F: far; S: small; $\mathbf{M}$ : medium; B: big

\begin{tabular}{l|l|l|l|l|l|l|l|l|l|l|l|l|l|l}
\hline \multirow{2}{*}{ Rule } & T & \multicolumn{9}{c}{ CD } & \multicolumn{3}{l|}{ ID } & \multicolumn{3}{l}{ Size } \\
& VC & C & M & W & VH & N & M & F & N & M & F & S & M & B \\
\hline T1 & $\geq 0.1$ & & & & & $\geq 0.5$ & & & & & & & & \\
\hline T2 & & $\geq 0.1$ & & & & $\geq 0.5$ & & & & & & & & \\
\hline T3 & & $\geq 0.3$ & & & & $\geq 0.5$ & & & & & & & & \\
\hline T4 & & $\geq 0.6$ & & & & $\geq 0.5$ & & & & & & & & \\
\hline T5 & & $\geq 0.8$ & & & & $\geq 0.5$ & & & & & & & & \\
\hline T6 & & & $\geq 0.8$ & & & $\geq 0.5$ & & & & & & & & \\
\hline T7 & & & $\geq 0.6$ & & & $\geq 0.5$ & & & & & & & & \\
\hline T8 & & & $\geq 0.3$ & & & $\geq 0.5$ & & & & & & & & \\
\hline T9 & & $\geq 0.1$ & & & & $\leq 0.5$ & & & & & & & & \\
\hline T10 & & & $\geq 0.6$ & & & & & & & & & & & \\
\hline T11 & & & $\geq 0.8$ & & & $\leq 0.5$ & & & & & & & & \\
\hline T12 & & $\geq 0.1$ & & & & & & & & & & & & \\
\hline T13 & & & $\geq 0.5$ & & & & & & & & & & & \\
\hline T14 & & & & $\geq 0.1$ & & & & & & & & & & \\
\hline T15 & & & $\geq 0.8$ & & & & & & & & & & & \\
\hline T16 & & & $\geq 0.1$ & & & & & & & & & & & \\
\hline T17 & & $\geq 0.1$ & & & & & & & $\geq 0.5$ & & & $\geq 0.4$ & & \\
\hline
\end{tabular}

labeling. Table 5 shows 17 basic knowledge rules for temperature images (MODISAqua-SST), and Table 4 shows the knowledge rules used for MOS labeling.

Again, temperature, near continent distance and near island distance are the most used descriptors. temperature images have a higher chromatic variability, and thus a large number of knowledge rules are required for MOS labeling. Automatic detection of MOS is prioritized, but some cases require manual processing.

\subsection{Labeling}

A total of 31 knowledge rules are able to label 365 MOS. Labeled MOS can be simple or merged. In Table 6 the number of simple and merged labeled regions are shown, indicating in each case the knowledge rules, as well as the origin (SeaWiFS, MODIS-Aqua-OC and MODIS-Aqua-SST, respectively). A total of 111 MOS for MODIS-Aqua-SST have been labeled (86 merged and 25 simple regions), and 158 MOS for SeaWiFS (131 merged and 27 simple regions). Finally, 96 MOS for MODISAqua-OC have been labeled ( 88 merged and 8 simple regions). 
Table 6 Result. MR: Merged Region; SR: Simple Region

\begin{tabular}{|c|c|c|c|c|c|c|c|c|}
\hline \multicolumn{9}{|l|}{ Result } \\
\hline SeaWiFS & MR & SR & MODIS-OC & MR & SR & MODIS-SST & MR & SR \\
\hline $\mathrm{RC} 1$ & 84 & 5 & $\mathrm{RC} 1$ & 26 & 3 & RT1 & 2 & 0 \\
\hline $\mathrm{RC} 2$ & 28 & 0 & $\mathrm{RC} 2$ & 19 & 0 & RT2 & 19 & 0 \\
\hline $\mathrm{RC} 3$ & 5 & 0 & $\mathrm{RC} 3$ & 29 & 2 & RT3 & 17 & 0 \\
\hline $\mathrm{RC} 4$ & 5 & 7 & $\mathrm{RC} 4$ & 4 & 0 & RT4 & 5 & 0 \\
\hline RC5 & 2 & 1 & RC5 & 1 & 0 & RT5 & 10 & 1 \\
\hline RC6 & 0 & 1 & RC6 & 2 & 0 & RT6 & 5 & 0 \\
\hline $\mathrm{RC7}$ & 1 & 0 & $\mathrm{RC} 7$ & 0 & 0 & RT7 & 2 & 0 \\
\hline $\mathrm{RC} 8$ & 5 & 1 & $\mathrm{RC} 8$ & 6 & 1 & RT8 & 18 & 0 \\
\hline RC9 & 0 & 4 & RC9 & 0 & 1 & RT9 & 2 & 0 \\
\hline $\mathrm{RC} 10$ & 1 & 3 & $\mathrm{RC} 10$ & 0 & 0 & RT10 & 2 & 0 \\
\hline $\mathrm{RC} 11$ & 0 & 2 & $\mathrm{RC} 11$ & 0 & 1 & RT11 & 0 & 5 \\
\hline $\mathrm{RC} 12$ & 0 & 3 & $\mathrm{RC} 12$ & 1 & 0 & RT12 & 0 & 1 \\
\hline & & & & & & RT13 & 1 & 0 \\
\hline & & & & & & RT14 & 0 & 1 \\
\hline & & & & & & RT15 & 0 & 1 \\
\hline & & & & & & RT16 & 2 & 4 \\
\hline & & & & & & RT17 & 1 & 0 \\
\hline & & & & & & RT18 & 0 & 10 \\
\hline & & & & & & RT19 & 0 & 2 \\
\hline
\end{tabular}

Table 7 Labeling result of SeaWiFS. Yes: ROI; No: No ROI

\begin{tabular}{l|l|l|l|l|l|l|l|l|l|l|l}
\hline \multirow{2}{*}{ Rule } & \multicolumn{2}{l}{ Upwelling } & \multicolumn{2}{l}{ Upwelling Filament } & \multicolumn{2}{l|}{ Coldeddy } & \multicolumn{2}{l|}{ Warmeddy } & \multicolumn{2}{l}{ Wake } \\
\cline { 2 - 13 } & No & Yes & No & Yes & No & Yes & No & Yes & No & Yes \\
\hline RC1 & 88 & 1 & & & & & & & & \\
\hline RC2 & 28 & 0 & & & & & & & & \\
\hline RC3 & 5 & 0 & & & & & & & & \\
\hline RC4 & & & 12 & 0 & & & & & & \\
\hline RC5 & & & 2 & 1 & & & & & & \\
\hline RC6 & & & 1 & 0 & & & & & & \\
\hline RC7 & & & 1 & 0 & & & & & & \\
\hline RC8 & & & 0 & 6 & & & & & & \\
\hline RC9 & & & & & & 0 & 4 & & & & \\
\hline RC10 & & & & & & & & 0 & 2 & & \\
\hline RC11 & & & & & & & & & & 0 & 3 \\
\hline RC12 & & & & & & & & & & \\
\hline
\end{tabular}


Table 8 Labeling result of MODIS-OC. Yes: ROI; No: No ROI

\begin{tabular}{l|l|l|l|l|l|l|l|l|l|l}
\hline \multirow{2}{*}{ Rule } & Upwelling & \multicolumn{2}{l}{ Upwelling Filament } & \multicolumn{2}{l}{ Cold eddy } & \multicolumn{2}{l}{ Warm eddy } & \multicolumn{2}{l}{ Wake } \\
\cline { 2 - 14 } & No & Yes & No & Yes & No & Yes & No & Yes & No & Yes \\
\hline RC1 & 29 & 0 & & & & & & & & \\
\hline RC2 & 19 & 0 & & & & & & & & \\
\hline RC3 & 30 & 1 & & & & & & & & \\
\hline RC4 & & & 4 & 0 & & & & & & \\
\hline RC5 & & & 1 & 0 & & & & & & \\
\hline RC6 & & & 1 & 1 & & & & & & \\
\hline RC7 & & & 0 & 0 & & & & & & \\
\hline RC8 & & & 2 & 5 & & & & & & \\
\hline RC9 & & & & & 0 & 1 & & & & \\
\hline RC10 & & & & & 0 & 0 & & & & \\
\hline RC11 & & & & & & & 0 & 1 & & \\
\hline RC12 & & & & & & & & & 0 & 1 \\
\hline
\end{tabular}

Some knowledge rules work fine for some specific structures, where automatic merging is enough (without ROI). In some other cases, manual merging is required. Tables 7, 8 and 9 specify the success of the rules in each type of MOS as well as whether the manual mode and ROI is required. From 158 labeled MOS in SeaWiFS images, manual mode (ROI) is used 27 times. Rules RC1 to RC3 facilitate upwelling detection (122), where only one time manual mode is required. Rules RC4 to RC8 are used for upwelling filaments (23). Manual mode is required 7 times in this case. Cold eddies ( 8 by rules RC 9 and RC10), warm eddies ( 2 by rule RC11) and wakes ( 3 by rule RC12) require manual mode. From 96 labeled MOS in MODIS-Aqua-OC images, manual mode (ROI) is used 10 times. Rules RC1 to RC3 facilitate upwelling detection (79), where only one time manual mode is required. Rules RC4 to RC8 are used for upwelling filaments (14). Manual mode is required 6 times in this case. Cold eddies ( 1 by rules RC9 and RC10), warm eddies ( 1 by rule RC11) and wakes ( 1 by rule RC12) require manual mode. From 111 labeled MOS in MODIS-Aqua-SST images, manual mode (ROI) is used 28 times. Rules RT1 to RT10 facilitate upwelling detection (83), where only one time manual mode is required. Rules RT11 to RT13 are used for upwelling filaments (7). Manual mode is required 6 times in this case. Cold eddies ( 1 by rules RT9 and RT14), warm eddies ( 1 by rule RT15) and wakes (11 by rules RT16 to RT19) require manual mode.

In summary, in most of the cases upwelling are easily detected in automatic mode (using a suitable tolerance level). Upwelling filaments, eddies and wakes are smaller in size, and more imprecise structures with respect to spectral and contextual properties, and thus in most of the cases they require manual mode to delimitate the area in which the region is found. 
Table 9 Labeling result of MODIS-. Yes: ROI; No: No ROI

\begin{tabular}{|c|c|c|c|c|c|c|c|c|c|c|}
\hline \multirow[t]{2}{*}{ Rule } & \multicolumn{2}{|c|}{ Upwelling } & \multicolumn{2}{|c|}{ Upwelling Filament } & \multicolumn{2}{|c|}{ Cold eddy } & \multicolumn{2}{|c|}{ Warmeddy } & \multicolumn{2}{|c|}{ Wake } \\
\hline & No & Yes & No & Yes & No & Yes & No & Yes & No & Yes \\
\hline RT1 & 2 & 0 & & & & & & & & \\
\hline RT2 & 19 & 0 & & & & & & & & \\
\hline RT3 & 17 & 0 & & & & & & & & \\
\hline RT4 & 5 & 0 & & & & & & & & \\
\hline RT5 & 11 & 0 & & & & & & & & \\
\hline RT6 & 5 & 0 & & & & & & & & \\
\hline RT7 & 2 & 0 & & & & & & & & \\
\hline RT8 & 18 & 0 & & & & & & & & \\
\hline RT9 & 1 & 1 & & & & & & & & \\
\hline RT10 & 2 & 0 & & & & & & & & \\
\hline RT11 & & & 0 & 5 & & & & & & \\
\hline RT12 & & & 0 & 1 & & & & & & \\
\hline RT13 & & & 1 & 0 & & & & & & \\
\hline RT14 & & & & & 0 & 1 & & & & \\
\hline RT15 & & & & & & & 0 & 1 & & \\
\hline RT16 & & & & & & & & & 0 & 6 \\
\hline RT17 & & & & & & & & & 0 & 1 \\
\hline RT18 & & & & & & & & & 0 & 10 \\
\hline RT19 & & & & & & & & & 0 & 2 \\
\hline
\end{tabular}

\section{Implementation}

In this section, we describe the implementation of the tool. First, the main elements of the user interface are described, and next the class and data modeling of the tool are shown. The tool has been implemented in Java, making use of the OpenCV $\mathrm{C}$ library for image processing. The Java code includes HDF processing with the HDF5 Java library, as well as the user interface implemented in Java Swing, and the business logic. Data access to SQL with JDBC from Java permits image and region data storing.

\subsection{User Interface}

The user interface has been designed to facilitate image processing and visualization as well as region merging and labeling. The selection of a color table is carried out from the user interface, using a text file containing the table items (see Fig. 5). To swap from a color clustering to another, color table files are stored in disc, and can 


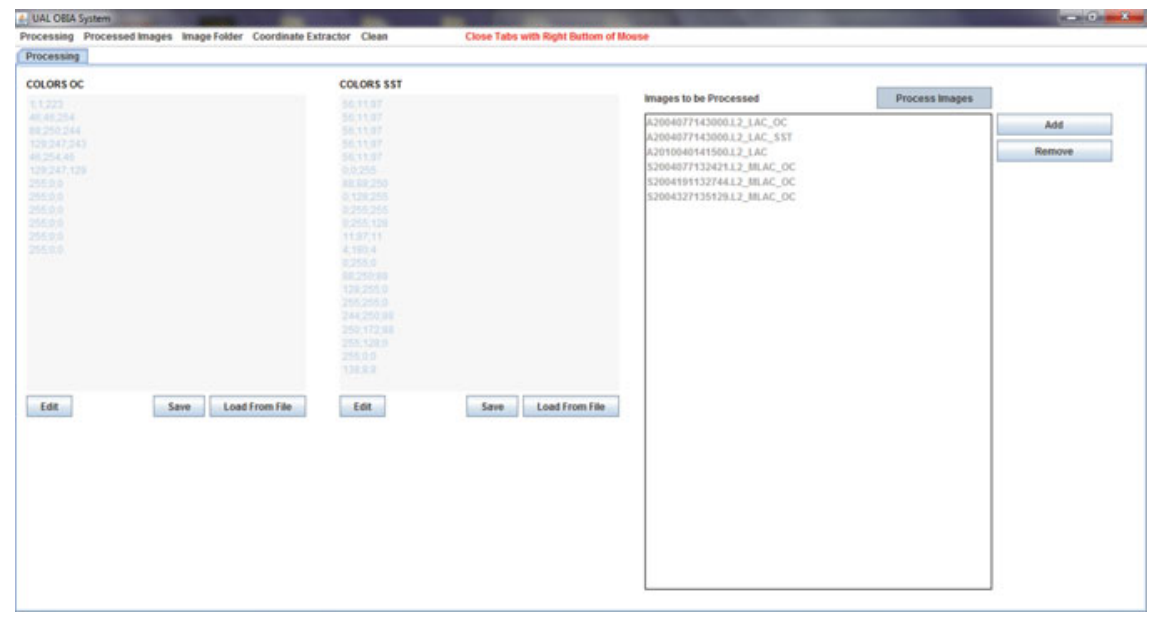

Fig. 5 Processing window

be selected any time. From this window, the oceanographer can select images to be processed, and can process images with the selected color table.

When segmentation is optimal (i.e., compact and well-contoured regions and complete MOS), the oceanographer can select the image to be labeled (see Fig. 6). From this window, images from a given sensor and earth area are shown. Selecting an image, the oceanographer can proceed to merge and label regions of the image.

Figure 7 shows the window from which knowledge rules are defined and executed. With the aim the user interface is equipped with a menu for selecting the fuzzy

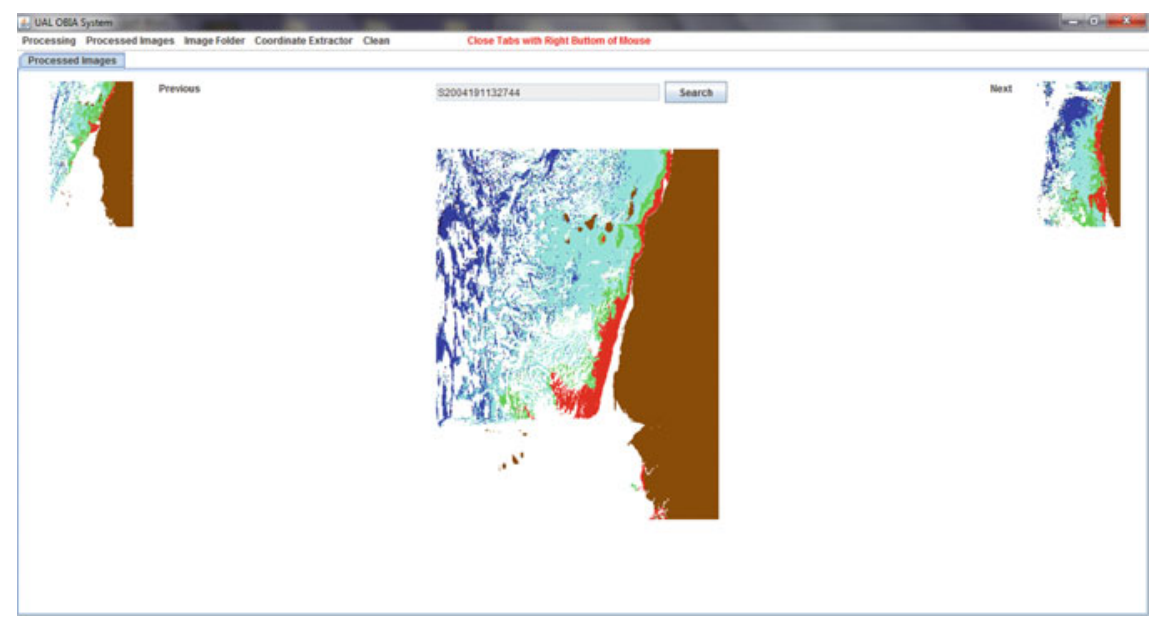

Fig. 6 Image selection window 


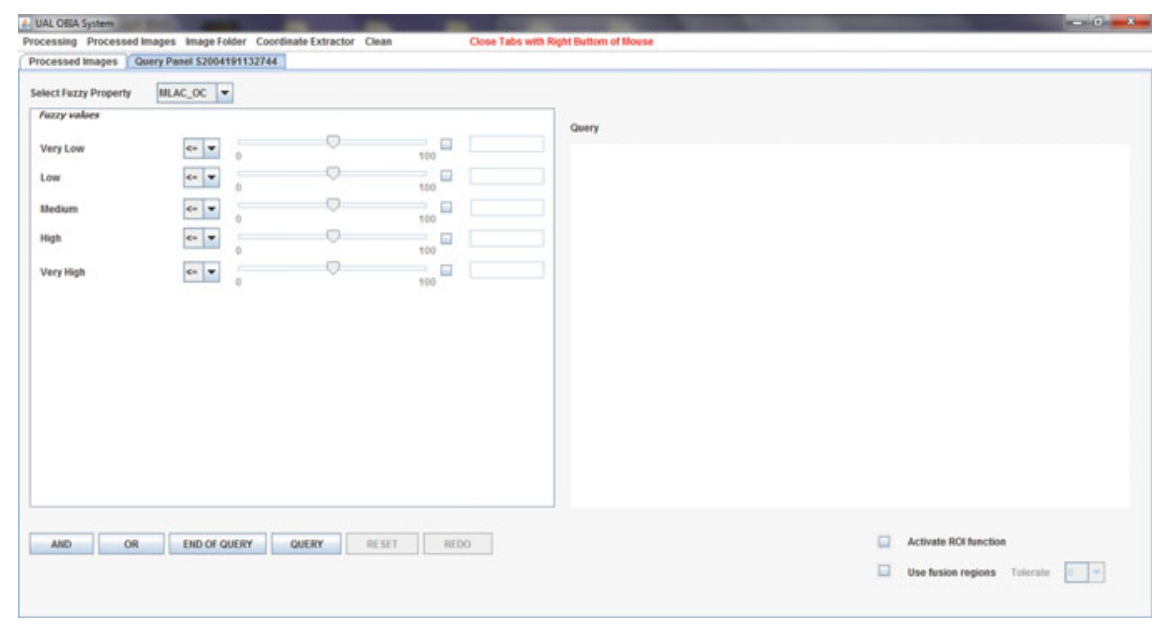

Fig. 7 Knowledge rule window

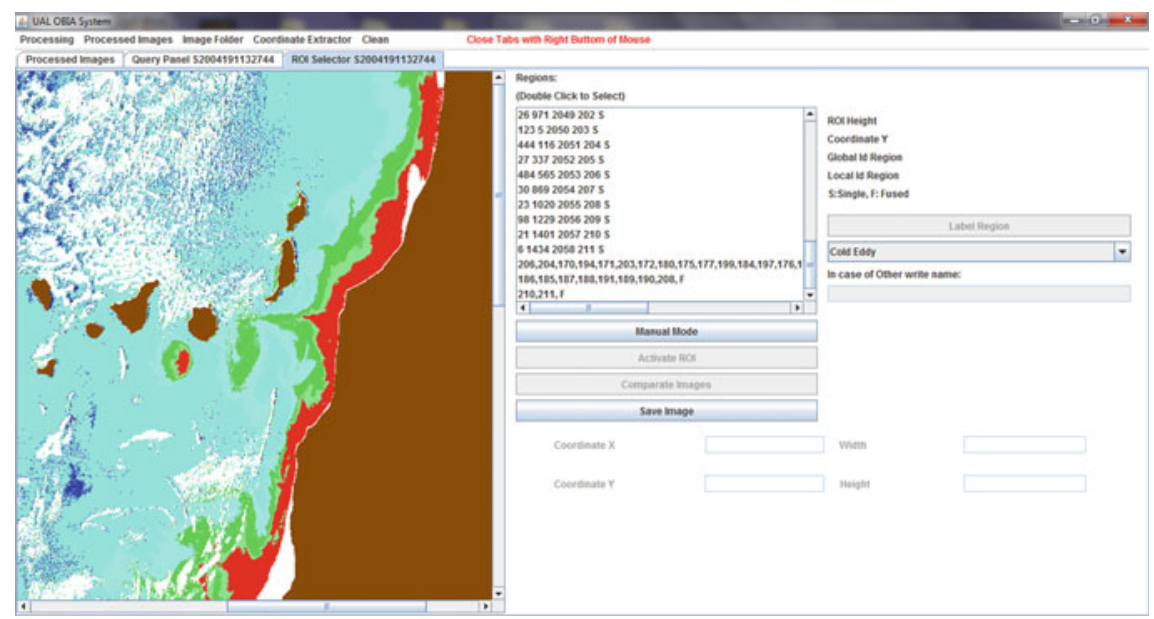

Fig. 8 Result window

descriptor (i.e., chlorophyll concentration, temperature, continent distance, island distance and size), the fuzzy value (i.e., in each case, the corresponding value: very high, high, medium, low, very low), and the range of the value (i.e., greater than, less than, equal, etc.,), to facilitate the definition of knowledge rules. ANDs and ORs connectives can be used as many as the user specifies. From this window, the oceanographer can select automatic merging of regions, and can select tolerance level. He/she can also activate automatic ROI.

The window used to list the results is shown in Fig. 8. In this example the knowledge rule Fuzzy_value_5 $\geq 0.25$ OR Fuzzy_value_3 $\geq 0.25$ has been used, where the 
Fuzzy_value attribute correspond to fuzzy values of chlorophyll (1-very low, 2-low, 3-medium, 4-high, 5-very high) and temperature (1-very cold, 2-cold, 3-temperate, 4-warm, 5-very warm). In this example regions of very high (i.e., Fuzzy_value_5) or medium (i.e., Fuzzy_value_3) chlorophyll concentration have to be found, typical of upwellings and cold eddies. The window shows the image, and a list of regions retrieved by the knowledge rule. For each listed region, the tool shows the height of the ROI (i.e., height of the Bounding box of the region), the Y coordinate in the image, the identifier in the database, and S/F, which means simple $(\mathrm{S})$ or fused $(\mathrm{F})$ (i.e., merged) region. In the case of merged regions, the tool shows the identifier of each single region taking part of the merged region. By double-click on any element of the list, the tool emphasizes in pink color the region to be easily localized. The knowledge rule returns an empty list if it is not suitable for the retrieval of regions. Otherwise, the oceanographer can select and label a region from the list. Additionally, the oceanographer can select manual mode. In manual mode the manual ROI (i.e., a rectangle) is activated, and the selected regions can be grouped. When regions are grouped, the oceanographer can also label. The tool automatically unifies the regions, and they are labeled by the oceanographer (button "Label Region"). For instance, for labeling the cold eddy in the south of Gran Canaria, a ROI is selected with regions of the area.

The tool also permits, from this window, to compare with previously labeled regions. The tool retrieves images with regions of similar properties, to assist in the identification of structures in the current image (see Fig. 9). In Fig. 9 the tool shows, on the left-hand side, the current image with the selected region in pink color (i.e., a cold eddy), and on the right-hand side, an image (a SeaWiFS scene from 2004-03-17) in which a wake has been previously labeled (with similar properties to the eddy).

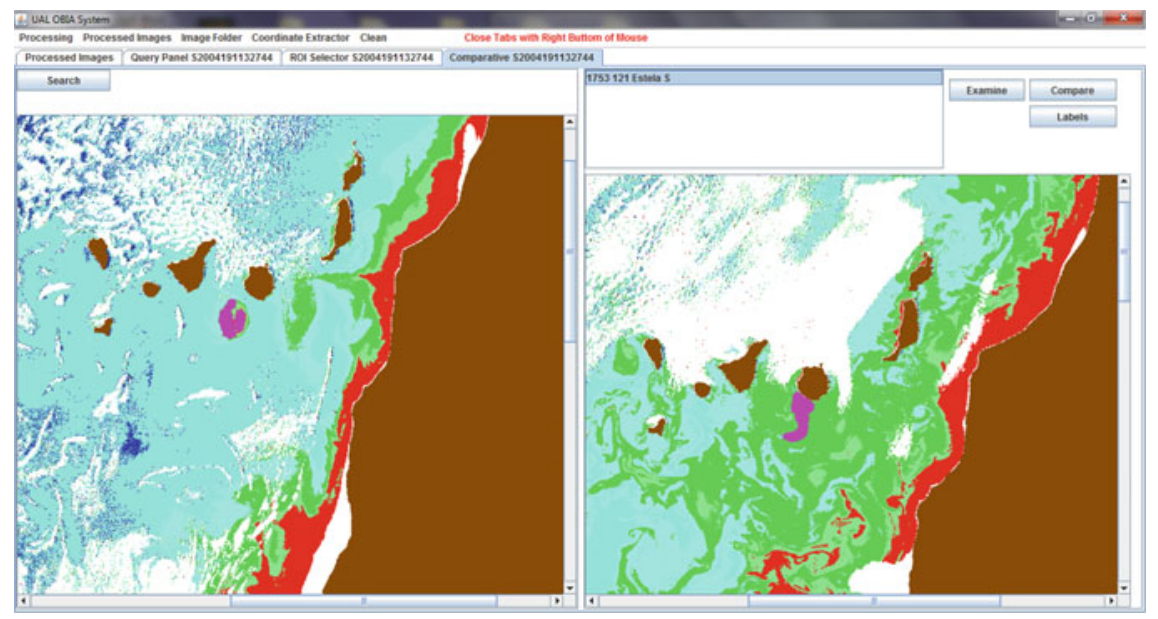

Fig. 9 image comparison window 
The tool facilitates to modify the knowledge rules any time, with the goal to refine them by selecting other fuzzy descriptors, by changing value range, by deselecting automatic merging mode, and by increasing/decreasing tolerance level.

\subsection{Class Modeling}

In this section, we describe the class modeling of the tool. With the aim to have a friendly, robust and extensible tool, UML (Unified Modeling Language) has been used for modeling. Class modeling is shown in Fig. 10. A modular and extensible structure has been modeled. The model consists of three main elements: the user interface, the business logic, and the data logic. The user interface has been implemented with frames and panels of the Java Swing library (they are stereotyped in Fig. 10).

The main frame is a menu from which the tasks of the workflow (see Fig. 2 of Sect. 3) can be executed. The oceanographer can go back any time to previous tasks. Several images and regions to label can be selected at the same time. The main panels are PanelProcessing, PanelFolder, PanelSelector, PanelQuery and PanelImages. PanelProcessing is in charge of color clustering, image selection for processing, and processing. PanelFolder shows the folder (original, gray scale, mask, etc.,) for image visualization. PanelSelector permits the selection of the image to be labeled. PanelQuery facilitates the edition and execution of knowledge rules. Finally, PanelImages is used for labeling and manual merging of regions, as well as for comparison of images. The business logic is implemented by the class interface, which is in charge of the connection between the application and the database, which is handled by the class Database. Database class implements the database access using the Java JDBC library.

\subsection{Data Modeling}

In this section, we describe the design of the database. The database model is described by an entity-relationship diagram and shown in Fig. 11. The main table is Region (on the right-hand side) and stores region data. On the left-hand side a similar table is used to store merged regions. The table stores region descriptors including:

1. Hu moments: $H u 1, \ldots, H u 7$ [41],

2. Maitra moments: $M M 1, \ldots, M M 6$ [42],

3. Tensorial moments: M00, M01, M02, M03, M10, M11, M12, M20, M21 and M30 [43],

4. Zernike moments: $M Z 1, \ldots, M Z 6, M P 1$ and $M P 2$ [44],

5. Perimeter, Area, Circularity, Eccentricity, MajorAxis, MinorAxis [45], CentroidX, CentroidY, Cirscumpcription and Orientation descriptors, 


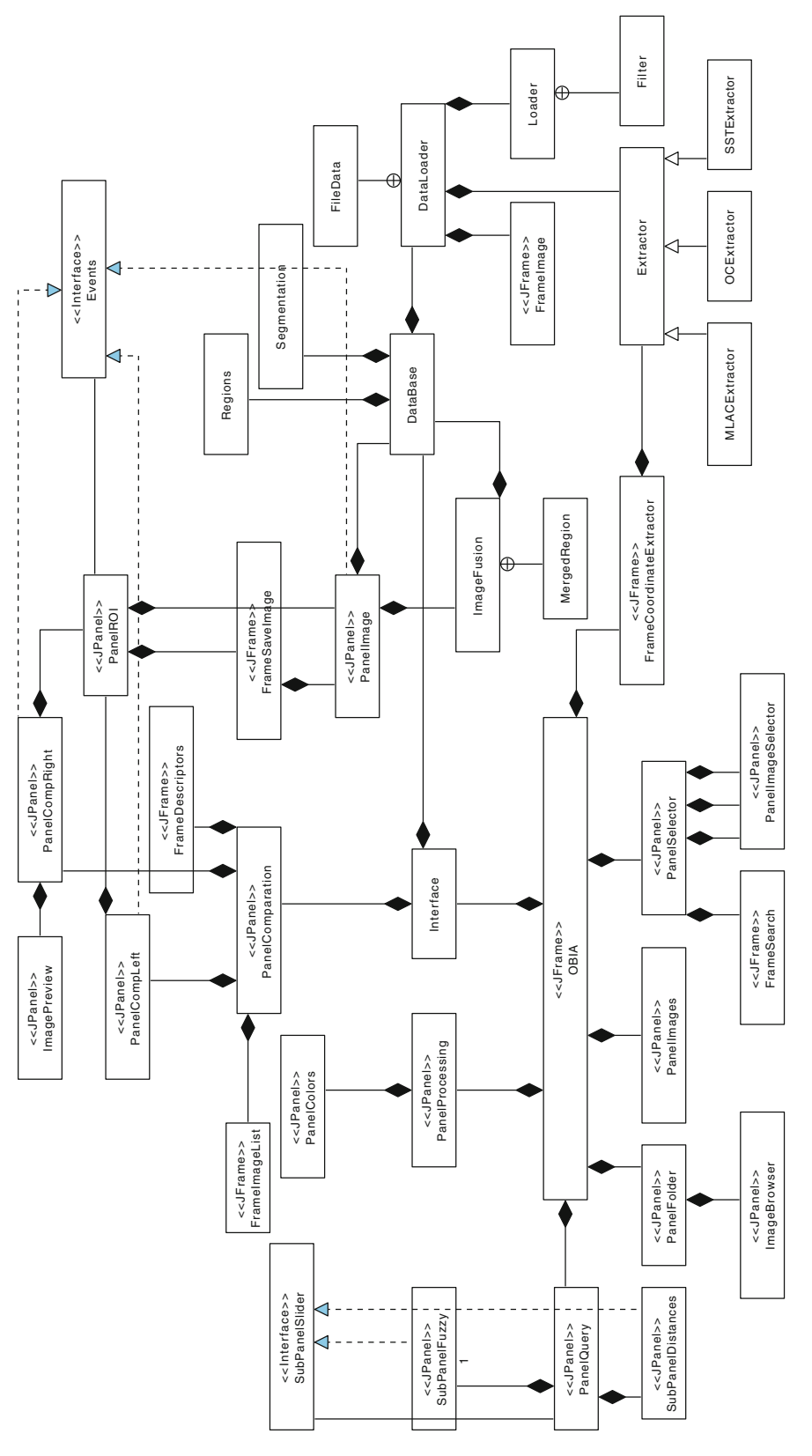

$: 0$
$\vdots 0$
0
0
0
0
0
0
0
0
0
00
$\vdots 00$ 


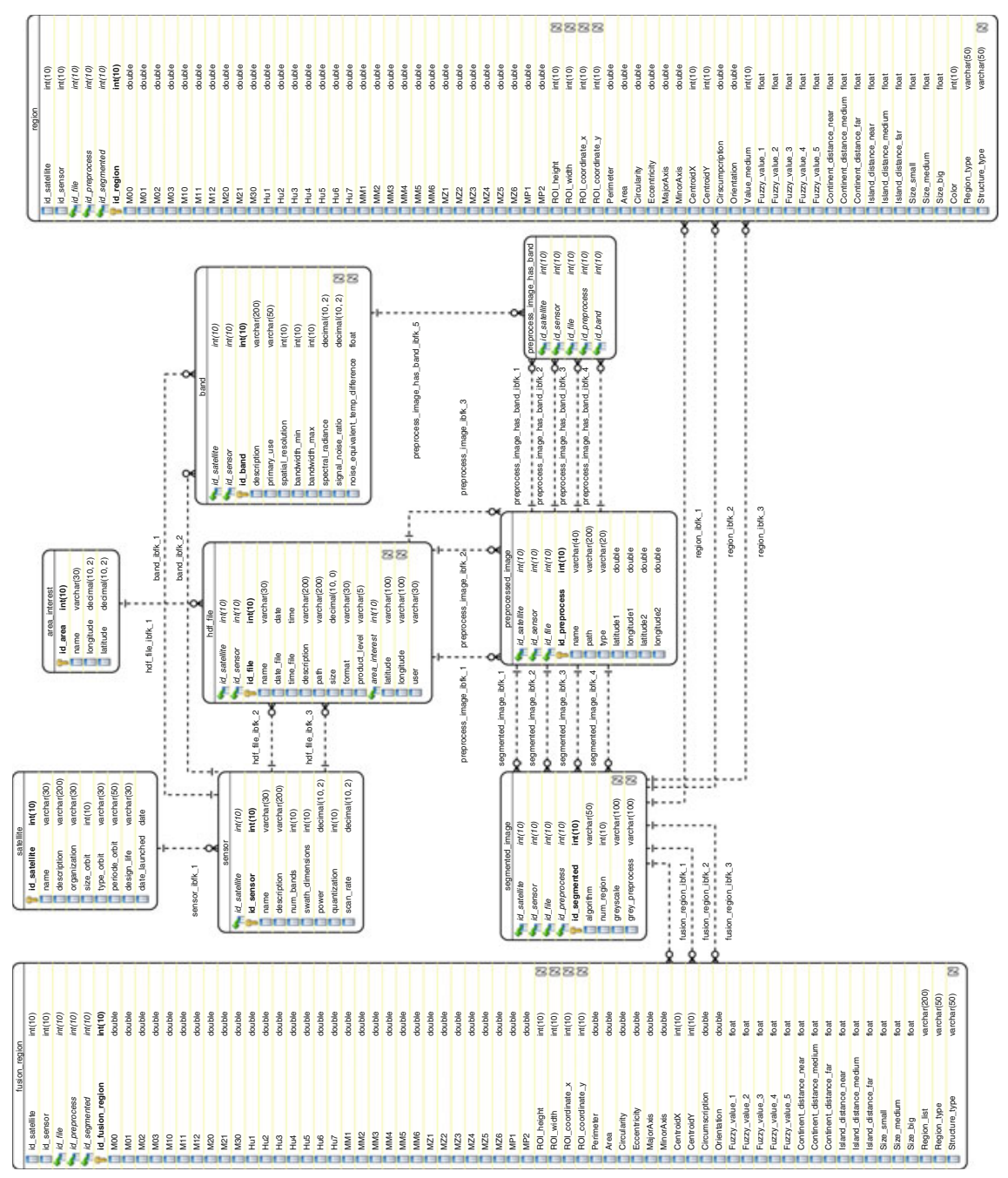


6. ROI descriptors (i.e., ROI_height, ROI_width, ROI_coordinate_X, ROI_coordinate_Y),

7. And the fuzzy values of size, island distance, chlorophyll concentration and temperature.

The data model also uses tables for storing satellite data (table Satellite), sensor data (table Sensor), as well as areas of interest (table Area_interest) and sensor bands (table Band). HDF files data are stored in the table $h d f$ file. Finally, segmented_image, preprocessed_image and preprocessed_image_has_band tables store data about segmented and preprocessed images.

\section{Conclusions and Future Work}

In this chapter we have described the implementation of a tool for the identification and labeling of MOS. We have described the workflow of the tool, the user interface, the class diagram, and the database. As part of the workflow we have described how to extract knowledge rules for the labeling of MOS. We have presented the results obtained with the tool from the analysis of images of the Canary Islands and North West African coast captured by the SeaWiFS and MODIS-AQUA sensors. A total of 31 knowledge rules (12 for images and 19 for temperature images) have been obtained, which are required to label 365 MOS. The tool is available for download in http://indalog.ual.es/obia.

The extraction of the knowledge rules took several months of trial and error. The goal was to label each image and a larger number of MOS in each image. The goal was also to have a reduced number of knowledge rules. In our opinion, oceanographers would find useful to have a small number of rules, with the aim to label MOS of the study area in reasonable time, even though they are novice users. We believe that a novice user would spend less than three minutes in labeling a MOS. An expert user would label an image in thirty seconds. temperature images are more difficult to label. It is due to that the ocean temperature in the time of image capture greatly affects the MOS identification and retrieval. This is the reason why the number of knowledge rules for temperature (19) is greater than for chlorophyll (12). In chlorophyll images, three knowledge rules are enough to label all the upwellings, while in temperature images ten rules are required. upwellings are cold water structures close to coast. Thus, upwelling are harder to label when the ocean temperature is colder.

We believe that the developed tool can help oceanographers in the identification and labeling of MOS, as well as in the definition of knowledge rules for their recognition. The tool assists oceanographers in the processing of a batch of images, their visualization, and their labeling, and is very helpful in the tedious task of labeling large image databases.

Our tool has be designed to work with images from the Canary Islands and North West African coast. It means that our tool has to be modified a little in order to accept images of other places of interest for oceanographers from the point of view 
of global climate and primary production. This is the case, for instance, of MOS in some areas of North Atlantic and North Pacific [13-15] which have similar ocean current and wind conditions to the current study area. On the other hand, with the aim to contribute to the study of the marine environment and the coastal resource management, we are now working on adapt our tool to the detection of trends and changes in chlorophyll concentration or temperature, by analyzing the morphology, movement, and evolution of MOS.

Acknowledgments This work was funded by the EU ERDF and the Spanish Ministry of Economy and Competitiveness (MINECO) under Projects TIN2013-41576-R, TIN2013-44742-C4-4-R and CGL2013-48202-C2-2-R, and the Andalusian Regional Government (Spain) under Project P10-TIC-6114. This work also received funding from the CEiA3 and CEIMAR consortiums.

\section{References}

1. Bakun, A.: Global climate change and intensification of coastal ocean upwelling. Science 247(4939), 198-201 (1990)

2. Change, I.P.O.C.: Climate change 2013: the physical science basis. Agenda 6(07), 333 (2013)

3. McGregor, H., Dima, M., Fischer, H., Mulitza, S.: Rapid 20th-century increase in coastal upwelling off northwest Africa. Science 315(5812), 637-639 (2007)

4. Gregg, W.W., Conkright, M.E., Ginoux, P., O'Reilly, J.E., Casey, N.W.: Ocean primary production and climate: global decadal changes. Geophys. Res. Lett 30(15), 1809 (2003)

5. Angel, M.V., Fasham, M.J.R.: Eddies and biological processes. Eddies in Marine Science, pp. 492-524. Springer, Berlin (1983)

6. Rubino, A.: Fluctuating mesoscale frontal features: structures and manifestations in the real ocean. Kumulative Habilitationsschrift, Universitat Hamburg (2005)

7. Birkhoff, G., et al.: Jets, Wakes, and Cavities. Elsevier, Amsterdam (2012)

8. Chelton, D.B., Schlax, M.G., Samelson, R.M., de Szoeke, R.A.: Global observations of large oceanic eddies. Geophys. Res. Lett. 34(15), L15606 (2007)

9. Robinson, I.S.: Ocean mesoscale features: upwelling and other phenomena. Discovering the Ocean from Space, pp. 159-193. Springer, Berlin (2010)

10. Schwartz, M.: Encyclopedia of Coastal Science. Springer Science \& Business Media, New York (2006)

11. Zhang, Z., Zhang, Y., Wang, W., Huang, R.X.: Universal structure of mesoscale eddies in the ocean. Geophys. Res. Lett. 40(14), 3677-3681 (2013)

12. Barton, E.D., Arístegui, J., Tett, P., Cantón, M., García-Braun, J., Hernández-León, S., Nykjaer, L., Almeida, C., Almunia, J., Ballesteros, S., Basterretxea, G., Escánez, J., García-Weil, L., Hernández-Guerra, A., López-Laatzen, F., Molina, R., Montero, M.F., Navarro-Pérez, E., Rodríguez, J.M., van Lenning, K., Vélez, H., Wild, K.: The transition zone of the Canary Current upwelling region. Progress Oceanogr. 41(4), 455-504 (1998)

13. Kersalé, M., Doglioli, A., Petrenko, A.: Sensitivity study of the generation of mesoscale eddies in a numerical model of Hawaii islands. Ocean Sci. 7(3), 277-291 (2011)

14. Lorenzo, E.D., Miller, A.J., Neilson, D.J., Cornuelle, B.D., Moisan, J.R.: Modelling observed California Current mesoscale eddies and the ecosystem response. Int. J. Remote Sens. 25(7-8), 1307-1312 (2004)

15. Lumpkin, C.F.: Eddies and currents of the Hawaiian Islands. Ph.D. thesis, University of Hawaii (1998)

16. Oke, P.R., Griffin, D.A.: The cold-core eddy and strong upwelling off the coast of New South Wales in early 2007. Deep Sea Res. Part II: Top. Stud. Oceanogr. 58(5), 574-591 (2011) 
17. Meunier, T., Barton, E.D., Barreiro, B., Torres, R.: Upwelling filaments off Cap Blanc: interaction of the NW African upwelling current and the Cape Verde frontal zone eddy field. J. Geophys. Res.: Oceans (1978-2012) 117, C8 (2012)

18. Tejera, A., García-Weil, L., Heywood, K., Cantón-Garbín, M.: Observations of oceanic mesoscale features and variability in the Canary Islands area from ERS-1 altimeter data, satellite infrared imagery and hydrographic measurements. Int. J. Remote Sens. 23(22), 4897-4916 (2002)

19. Sangra, P., Pelegrí, J., Hernández-Guerra, A., Arregui, I., Martín, J., Marrero-Díaz, A., Martínez, A., Ratsimandresy, A., Rodríguez-Santana, A.: Life history of an anticyclonic eddy. J. Geophys. Res. 110(C3), C03, 021 (2005)

20. Arístegui, J., Sangra, P., Hernández-León, S., Cantón, M., Hernández-Guerra, A., Kerling, J.: Island-induced eddies in the Canary islands. Deep Sea Res. Part I: Oceanogr. Res. Pap. 41(10), 1509-1525 (1994)

21. Baatz, M., Hoffmann, C., Willhauck, G.: Progressing from object-based to object-oriented image analysis. Object-Based Image Analysis, pp. 29-42. Springer, Berlin (2008)

22. Musci, M., Feitosa, R.Q., Costa, G.A.: An object-based image analysis approach based on independent segmentations. In: Urban Remote Sensing Event (JURSE), 2013 Joint, pp. 275278. IEEE (2013)

23. Drăguţ, L., Blaschke, T.: Automated classification of landform elements using object-based image analysis. Geomorphology 81(3), 330-344 (2006)

24. Jovanovic, D., Govedarica, M., Dordevic, I., Pajic, V.: Object based image analysis in forestry change detection. In: 2010 8th International Symposium on Intelligent Systems and Informatics (SISY), pp. 231-236. IEEE (2010)

25. Rastner, P., Bolch, T., Notarnicola, C., Paul, F.: A comparison of pixel-and object-based glacier classification with optical satellite images. IEEE J. Sel. Top. Appl. Earth Obs. Remote Sens. 7(3), 853-862 (2014)

26. Ko, B., Byun, H.: Frip: a region-based image retrieval tool using automatic image segmentation and stepwise Boolean AND matching. IEEE Trans. Multimed. 7(1), 105-113 (2005)

27. Shrivastava, N., Tyagi, V.: A review of roi image retrieval techniques. In: Proceedings of the 3rd International Conference on Frontiers of Intelligent Computing: Theory and Applications (FICTA) 2014, pp. 509-520. Springer, Berlin (2015)

28. Vidal-Fernández, E., Piedra, J.A., Almendros-Jiménez, J.M., Cantón, M.: A location based approach to classification of mesoscale oceanic structures in SeaWiFS and MODIS-Aqua images from the North West Africa Area. Int. J. Remote Sens. 36(24), 6135-6159 (2015)

29. Vidal-Fernández, E., Piedra, J.A., Almendros-Jiménez, J.M., Cantón, M.: OBIA system for identifying mesoscale oceanic structures in SeaWiFS and MODIS-aqua images. IEEE J. Sel. Top. Appl. Earth Obs. Remote Sens. 8(3), 1256-1265 (2015)

30. Almendros-Jiménez, J.M., Domene, L., Piedra-Fernández, J.A.: A framework for ocean satellite image classification based on ontologies. IEEE J. Sel. Top. Appl. Earth Obs. Remote Sens. 6(2), 1048-1063 (2013)

31. Piedra-Fernandez, J.A., Cantón-Garbín, M., Wang, J.Z.: Feature selection in AVHRR ocean satellite images by means of filter methods. IEEE Trans. Geosci. Remote Sens. 48(12), 41934203 (2010)

32. Piedra-Fernández, J.A., Ortega, G., Wang, J.Z., Cantón-Garbín, M.: Fuzzy content-based image retrieval for oceanic remote sensing. IEEE Trans. Geosci. Remote Sens. 52(9), 5422-5431 (2014)

33. NASA: Ocean Color Web. http://oceancolor.gsfc.nasa.gov (2013)

34. Liu, Z., Hou, Y.: Kuroshio front in the East China Sea from Satellite SST and remote sensing data. IEEE Geosci. Remote Sens. Lett. 9(3), 517-520 (2012)

35. Marcello, J., Marques, F., Eugenio, F.: Automatic tool for the precise detection of upwelling and filaments in remote sensing imagery. IEEE Trans. Geosci. Remote Sens. 43(7), 1605-1616 (2005)

36. Mityagina, M., Lavrova, O.: Dynamic phenomena in the coastal waters of the north-eastern black sea retrieved from satellite data. In: IEEE International Geoscience and Remote Sensing Symposium, 2008. IGARSS 2008, vol. 2, pp. II-347. IEEE (2008) 
37. Patel, S., Balasubramanian, R., Gangopadhyay, A.: Automatic detection of oceanic eddies in SeaWiFS-derived color images using neural networks and shape analysis. Proc. IEEE IGARSS 2, II-835-II-838 (2008)

38. Xiao, B., Hu, S., Qiang, X.: Research on the ocean primary production pattern based remote sensing. In: 2010 International Conference on Audio Language and Image Processing (ICALIP), pp. 1543-1546. IEEE (2010)

39. Sathyendranath, S., Brewin, B., Mueller, D., Doerffer, R., Krasemann, H., Mélin, F., Brockmann, C., Fomferra, N., Peters, M., Grant, M., et al.: Ocean colour climate change initiativeapproach and initial results. In: IEEE International and Geoscience and Remote Sensing Symposium (IGARSS), pp. 2024-2027. IEEE (2012)

40. Saulquin, B., Gohin, F., Garrello, R.: Regional objective analysis for merging high-resolution MERIS, MODIS/Aqua, and SeaWiFS chlorophyll-a data from 1998 to 2008 on the European Atlantic shelf. IEEE Trans. Geosci. Remote Sens. 49(1), 143-154 (2011)

41. Hu, M.K.: Visual pattern recognition by moment invariants. IRE Trans. Inf. Theory $\mathbf{8}(2), 179$ 187 (1962)

42. Maitra, S.: Moment invariants. Proc. IEEE 67, 697-699 (1979)

43. Galvez, J.M., Cantón, M.: Normalization and shape recognition of three-dimensional objects by 3d moments. Pattern Recognit. 26(5), 667-681 (1993)

44. Teague, M.R.: Image analysis via the general theory of moments. J. Opt. Soc. Am. 70, 920-930 (1980)

45. Zunic, J., Sladoje, N.: Efficiency of characterizing ellipses and ellipsoids by discrete moments. IEEE Trans. Pattern Anal. Mach. Intell. 22(4), 407-414 (2000) 